\title{
High scotopic/photopic ratio white-light-emitting diodes integrated with semiconductor nanophosphors of colloidal quantum dots
}

\author{
Sedat Nizamoglu, ${ }^{1}$ Talha Erdem, ${ }^{1}$ and Hilmi Volkan Demir ${ }^{1,2,3,4}$ \\ ${ }^{1}$ Department of Electrical and Electronics Engineering, Department of Physics, and UNAM-Institute \\ of Materials Science and Nanotechnology, Bilkent University, Ankara, Turkey 06800 \\ ${ }^{2}$ School of Electrical and Electronic Engineering, \\ School of Physical and Mathematical Sciences, Nanyang Technological University, Singapore 639798 \\ ${ }^{3}$ e-mail: hvdemir@ntu.edu.sg \\ ${ }^{4}$ e-mail: olkan@bilkent.edu.tr
}

Received December 2, 2010; revised February 5, 2011; accepted February 8, 2011; posted March 10, 2011 (Doc. ID 139082); published May 13, 2011

\begin{abstract}
We propose and demonstrate single-chip white-light-emitting diodes (WLEDs) integrated with semiconductor nanophosphors of colloidal quantum dots for high scotopic/photopic (S/P) ratio. These color conversion WLEDs achieve S/P ratios over 3.00, which exceeds the current limit of 2.50 in common lighting technologies, while sustaining sufficient levels of color rendering index. () 2011 Optical Society of America

OCIS codes: $160.4236,230.3670,250.5230$.
\end{abstract}

Experiments supported by the U.S. Department of Energy indicate that rod photoreceptors, which are considered to be responsible for scotopic (dark adapted) vision, may also affect photopic (photon adapted) vision [1]. These studies argue that the variation of pupil size strongly follows a scotopic-like spectrum at interior lighting power levels [2-6]. According to these studies, it could therefore be possible to improve vision and lighting energy efficiency by accounting for the role of these rods, for example, in designing light-emitting diodes (LEDs). However, today's light sources are commonly specified by photopic vision. For solid state lighting (SSL), the role of rod activity in vision has not been studied in LED chips.

The figure of merit showing the rod activity under the illumination of a light source is quantified in terms of the ratio of eye response to the light source under the scotopic conditions to that in the photopic case, referred to as the scotopic/photopic (S/P) ratio of the source [7]. This difference in the scotopic and photopic luminous efficacy of the source results from the shift of eye sensitivity function peaking at $555 \mathrm{~nm}$ (under photopic lighting conditions) to $507 \mathrm{~nm}$ (under scotopic lighting conditions), also known as the Purkinje shift. According to this theory, if there are two light sources emitting at the same optical power, the one exhibiting higher $\mathrm{S} / \mathrm{P}$ ratio is predicted to yield better perceived brightness along with better visual acuity [2]. This means that light sources with higher S/P ratios may potentially provide the equivalent levels of perceived brightness and visual acuity at a lower output power level. High S/P ratio, which provides higher correlated color temperature (CCT), yields relatively smaller pupils at a given photopic light level so that the object light rays are collected more at the central region of the eye, which may possibly result in improved optical vision, according to [8]. Therefore, in that case, this may potentially provide means for energy saving. There are still ongoing investigations and discussions on the possible effects of the S/P ratio. Regardless of the extent of their effects under debate, looking from the device perspective, it is not straightforward to make high S/P ratio light sources with sufficient level of color rendering, which enables color differentiation. There exists a trade-off between S/P ratio and color rendering index (CRI). This trend, which is observable for various light sources, makes it challenging to make both the S/P ratio and the CRI high at the same time.

In terms of the S/P ratio and the CRI, the performance of current lighting technologies is typically limited [9,10]. The $\mathrm{S} / \mathrm{P}$ ratios of common white-light sources range from 0.8 to 2.5 [10]. For example, incandescent light bulbs have a poor $\mathrm{S} / \mathrm{P}$ ratio of 1.41 despite featuring a perfect CRI of 100. As for today's commercially available SSL sources, among the most widely used ones are colorconversion LEDs that integrate yellow yttrium aluminum garnet phosphors; these white LEDs also exhibit poor S/P ratios typically ranging from 1.68 to 2.38 [11,12]. To date, single-chip light sources achieving high $\overline{\mathrm{S} / \mathrm{P}}$ ratios have not been demonstrated or realized. Different phosphors may in principle be integrated to improve a certain photometric property (e.g., the S/P ratio or the CRI), but this may come at the cost of reducing performance levels of other photometric parameters (e.g., a decreased luminous efficacy depending on their emission linewidths).

As an alternative luminophor, for future white LEDs, semiconductor nanocrystal (NC) quantum dots are strong candidates with their size tuneable and narrow emission nature, also accompanied with reasonably large photoluminescence quantum efficiency [13-18]. Therefore, NCs have recently been exploited for various color conversion LED applications [19-27]. Especially their color tuning capability allows for adjustable white-light generation using combinations of $\mathrm{NC}$ emitters (e.g., CdSe/ZnS core-shell NCs) integrated on InGaN/GaN LEDs [27]. In these previous studies using NCs or others (e.g., using four-color mixing of polychromatic lamps [28]), none of the reported white LEDs features a high $\mathrm{S} / \mathrm{P}$ ratio.

For the purpose of obtaining a high $\mathrm{S} / \mathrm{P}$ ratio with a reasonable CRI, our design strategy relies on mimicking 
blackbody radiators with high color temperatures. This is because such high temperature blackbody radiators have dominantly blue-cyan emission meaning that their CCT is high, while also characteristically exhibiting a high CRI. Therefore, the optical spectrum design with dominant blue-cyan emission can be carefully adjusted to use appropriate combinations of $\mathrm{NC}$ emitters to obtain a similar spectrum of these blackbody radiators with high $\mathrm{S} / \mathrm{P}$ ratios and CRIs. The operating principle of these hybrid NC LEDs relies on using the combined electroluminescence of LEDs and photoluminescence of NC color convertors. The fabrication details of our blue LED are explained in [27]. Since the blue and cyan emissions are required to be dominant as in the case of blackbody radiators with high CCTs, we employ a blue-emitting LED and cyan-emitting $\mathrm{NC}$ appropriately with strong enough color components iteratively determined to increase sufficiently the color temperature and make the $\mathrm{S} / \mathrm{P}$ ratio high. On the other hand, the green, yellow, and red NC emitters are strategically chosen to balance the blue and cyan emission for white-light generation.

For a proof-of-concept demonstration of such spectrally enhancing NC based white LEDs, we incorporate cyan-, green-, yellow-, and red-emitting CdSe/ZnS core/ shell NCs with the photoluminescence peaks of $\lambda_{\mathrm{PL}}=$ $490,540,580$, and $620 \mathrm{~nm}$ in toluene solution, respectively, in layers integrated directly on our blue InGaN/ GaN LED chips with the electroluminescence peak of $\lambda_{\mathrm{EL}}=452 \mathrm{~nm}$ by making their close-packed films through evaporating the excess toluene solvent. For our hybrid device, we integrate $119.7 \mathrm{nmol}$ cyan-, $4.652 \mathrm{nmol}$ green-, $0.990 \mathrm{nmol}$ yellow-, and $0.158 \mathrm{nmol}$ red-emitting NCs on the blue LED, and the total thickness of the color converting layer is approximately $50 \mu \mathrm{m}$. We experimentally obtain the white-light generating hybrid LED driven at various current injection levels at room temperature as shown in Fig. 1. The luminescence of this hybrid LED increases, as the injected current increases in this regime, leading to the following photometric properties at $25 \mathrm{~mA}$ at room temperature: tristimulus coordinates $(x, y)=$ $(0.251,0.246)$, luminous efficacy of optical radiation $(\mathrm{LER})=266.83 \mathrm{~lm} /$ Wopt $, \mathrm{S} / \mathrm{P}=3.04, \mathrm{CRI}=71.7$, and $\mathrm{CCT}=45 \mathrm{kK}$. This white-light spectrum satisfies both of

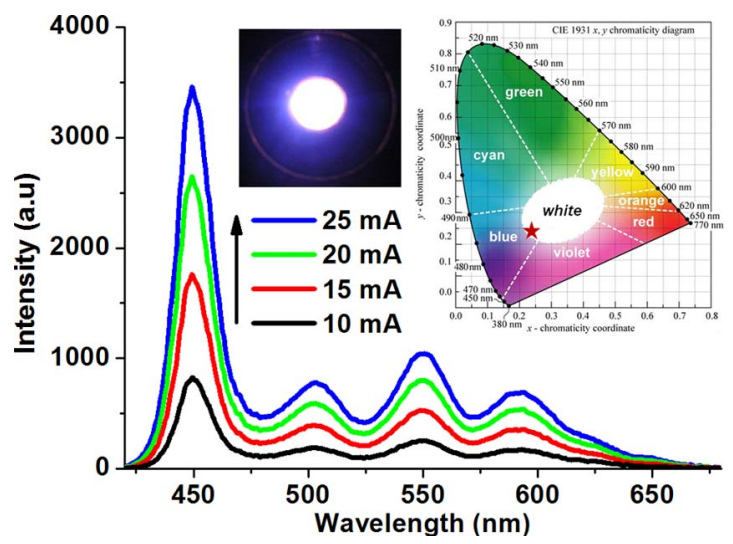

Fig. 1. (Color online) Emission spectra of our NC integrated white LED achieving a high S/P ratio of 3.05 at a CRI of 71.0 under various current injection levels at room temperature, along with its $(x, y)$ tristimulus coordinates on CIE 1931 chromaticity diagram and a photograph presented in the insets.

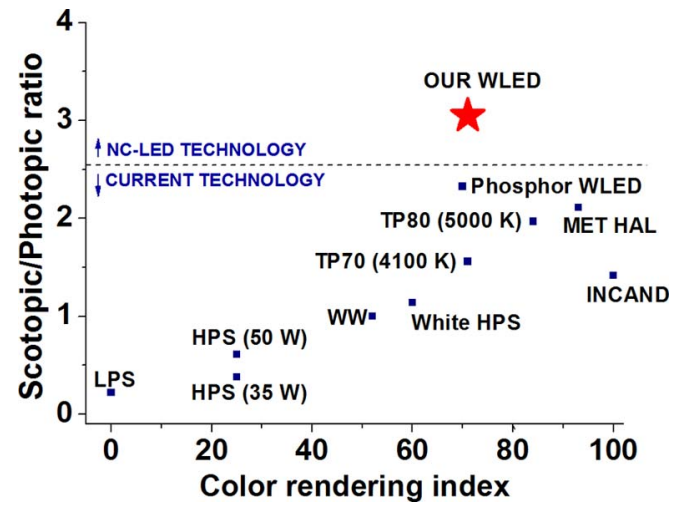

Fig. 2. (Color online) S/P ratio versus CRI given for our NCbased single-chip white LED, also compared with common lighting technologies [2] (including, LPS, low pressure sodium; HPS, high pressure sodium; WW, warm white fluorescent; TP70, triphosphor fluorescent $[70<\mathrm{CRI}<80]$; TP80, triphosphor fluorescent [ $80<C \mathrm{RI}<90]$; INCAND, incandescent light bulb; MET HAL, metal halide [Thal/Dys/Hol] [10], and also a typical yellow phosphor based WLED [9]]).

the figure of merits simultaneously for scotopic enhancement and reasonable color rendering for the first time, by achieving an $\mathrm{S} / \mathrm{P}$ ratio $>2.50$ at a $\mathrm{CRI}$ of 71.7 . Furthermore, the operating point of our white-light-emitting diodes (WLEDs) is expectedly close to the blue and white color intersection meaning that this WLED achieves a sufficiently cool white tint to increase the $\mathrm{S} / \mathrm{P}$ ratio.

At such elevated color temperatures, CRI may, however, not reflect the actual color rendering performance, thus we check our CRI with color quality scale (CQS) as well [29-31]. Our LED exhibits a CQS of 70.3, which confirms the color rendering strength of our LED with its close value to its CRI. By comparing our WLED against those of a typical phosphor-based white LED operating at the same optical power with an average $\mathrm{S} / \mathrm{P}$ ratio of $2.03(=[1.68+2.38] / 2)$, our NC luminophors is predicted to provide a $22.5 \%$ brighter view and a $50 \%$ better nighttime vision [32]. Such strong spectral enhancement is possible using emitters as a result of their conveniently size tuneable and precisely controllable emission thanks to the quantum size effect. Figure 2 also compares this NC-based white LED against different light sources available in common lighting technologies including phosphor-based white LEDs, along with their references. For example, the standard daylight source $\mathrm{D} 65$ yields an $\mathrm{S} / \mathrm{P}$ ratio of 2.47 [10]. As clearly observed in Fig. 2 , all common light sources lie below the $\mathrm{S} / \mathrm{P}$ ratio barrier of 2.50, and our NC-integrated source exceeds this barrier. This shows that white-light generation using $\mathrm{NC}$ luminophors outperforms current lighting technologies in terms of $\mathrm{S} / \mathrm{P}$ vision while achieving sufficiently high color rendering.

Table 1. Amounts of Integrated NCs Emitting at Different Colors (given in nmol) for Samples $A, B$, and $C$

\begin{tabular}{crccc}
\hline Samples & Cyan & Green & Yellow & Red \\
\hline A & 239.4 & 2.326 & 0.247 & 0.052 \\
B & 59.8 & 2.326 & 0.495 & 0.158 \\
C & 119.7 & 2.326 & 0.495 & 0.158 \\
\hline
\end{tabular}




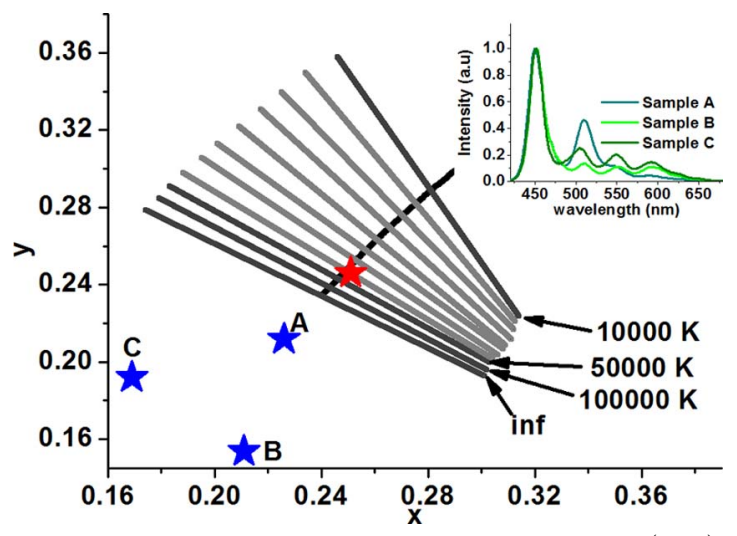

Fig. 3. (Color online) Lines of constant CCT on the $(x, y)$ chromaticity diagram with the emission spectra of Samples A, B, and $\mathrm{C}$. The red star is the $(x, y)$ tristimulus coordinates of the white LED shown in Fig. 1 .

We further investigate the $\mathrm{S} / \mathrm{P}$ ratio of other $\mathrm{NC}$ hybridized LEDs (dubbed Samples A, B, and C). Table 1 summarizes the integrated amount of each NC color component in these samples. Their resulting emission spectra and $(x, y)$ tristimulus coordinates are shown in Fig. 3 , and their optical properties are presented in Table 2. Sample A exhibits an $\mathrm{S} / \mathrm{P}$ ratio of 3.75 , and when we further enhance the blue emission in the spectrum, we achieve an increased S/P ratio of 4.72 for Sample B and of 5.15 for Sample C. Therefore, via increasing the blue content of the emission spectrum, LEDs with a higher S/P ratio can be obtained. However, since the $(x, y)$ tristimulus coordinates of these samples are then out of the chromaticity region spanned by the CCT isocurves as depicted in Fig. 3 , their CCTs cannot be defined. Thus, we cannot cite CRI values for these sources because we cannot utilize a reference spectrum to calculate their CRI. On the other hand, while falling outside the defined region of CCT, these LEDs are very close in spectral content to the LED design whose spectra are shown in Fig. 1. Therefore, Samples A-C are also expected to provide reasonable color rendering in practice, although their CRI is not technically definable.

In conclusion, this work demonstrated the first proposal and demonstration of scotopically enhancing singlechip white LEDs with NC luminophors to achieve a S/P ratio exceeding the common barrier of 2.50. These photometric properties show that these devices outperform common lighting technologies in terms of $\mathrm{S} / \mathrm{P}$ vision.

We acknowledge financial support from the National Research Foundation (NRF-RF-2009-09), European Science Foundation-European Young Investigator Awards, European Commission Framework Program (EC FP7 N4E NoE), Turkish Academy of SciencesYoung Scientists Award Programme, Scientific and Technological Research Council of Turkey-Department

Table 2. Photometric Properties of Samples A, B, and C

\begin{tabular}{ccccc}
\hline Samples & S/P Ratio & LER & $x$ & $y$ \\
\hline A & 3.75 & 228.24 & 0.226 & 0.212 \\
B & 4.72 & 178.48 & 0.211 & 0.154 \\
C & 5.15 & 201.04 & 0.169 & 0.192 \\
\hline
\end{tabular}

of Scientist Support, and Scientific and Technological Research Council of Turkey (107E088, 109E002, 109E004, and 110E010).

\section{References}

1. Pacific Northwest National Laboratory, "Spectrally enhanced lighting program implementation for energy savings: field evaluation," Tech Rep. PNNL-15784 (Pacific Northwest National Laboratory, 2006).

2. S. M. Berman, J. Illumin. Eng. Soc. 21, 1 (1992).

3. S. M. Berman, G. Fein, D. L. Jewett, and F. Ashford, J. Illumin. Eng. Soc. 22, 150 (1993).

4. S. M. Berman, G. Fein, D. L. Jewett, B. R. Benson, T. M. Law, and A. W. Myers, J. Illumin. Eng. Soc. 25, 51 (1996).

5. S. M. Berman, G. Fein, D. L. Jewett, and F. Ashford, J. Illumin. Eng. Soc. 23, 123 (1994).

6. S. M. Berman, G. Fein, D. L. Jewett, B. R. Benson, T. M. Law, A. W. Myers, and M. A. Bullimore, J. Illumin. Eng. Soc. 25, 42 (1996).

7. Commission Internationale de l'Eclairage, "Recommended system for mesopic photometry based on visual performance," Tech. Rep. 191:2010 (Commission Internationale de l'Eclairage Central Bureau, 2010).

8. S. M. Berman, M. Navvab, M. J. Martin, J. Sheedy, and W. Tithof, Light. Res. Technol. 38, 41 (2006).

9. J. Y. Tsao, IEEE Circuits Devices Mag. 20, 28 (2004).

10. J. A. Borton and K. A. Daley, IEEE Ind. Appl. Mag. 3, 54 (1997).

11. J. Van Derlofske, J. D. Bullough, and J. Watkinson, "Spectral effects of LED forward lighting," Tech Rep. TLA 2005-02 (RPI Lighting Research Center, 2005).

12. E. F. Schubert, Light Emitting Diode (Cambridge University, 2006).

13. A. P. Alivisatos, Science 271, 933 (1996).

14. D. J. Norris and M. G. Bawendi, Phys. Rev. B 53, 16338(1996).

15. V. I. Klimov, Semiconductor and Metal Nanocrystals: Synthesis and Electronic and Optical Properties (Marcel Dekker, 2003).

16. S. V. Gaponenko, Optical Properties of Semiconductor Nanocrystals (Cambridge University, 1998).

17. V. I. Klimov, A. A. Mikhailovsky, D. W. McBranch, C. A. Leatherdale, and M. G. Bawendi, Science 287, 1011 (2000).

18. M. Acherman, M. A. Petruska, S. Kos, D. L. Smith, D. D. Koleske, and V. I. Klimov, Nature 429, 642 (2004).

19. U. Banin, Nat. Photon. 2, 209 (2008).

20. J. M. Caruge, J. E. Halpert, V. Wood, V. Bulovic, and M. G. Bawendi, Nat. Photon. 2, 247 (2008).

21. Q. Sun, Y. A. Wang, L. S. Li, D. Wang, T. Zhu, J. Xu, C. Yang, and Y. Li, Nat. Photon. 1, 717 (2007).

22. S. Nizamoglu, G. Zengin, and H. V. Demir, Appl. Phys. Lett. 92, 031102 (2008).

23. P. O. Anikeeva, J. E. Halpert, M. G. Bawendi, and V. Bulovic, Nano Lett. 7, 2196 (2007).

24. N. Tessler, V. Medvedev, M. Kazes, S. Kan, and U. Banin, Science 295, 1506 (2002).

25. S. Nizamoglu, E. Sari, J.-H. Baek, I.-H. Lee, and H. V. Demir, New J. Phys. 10, 023026 (2008).

26. B. Guilhabert, D. Elfström, A. J. C. Kuehne, D. Massoubre, H. X. Zhang, S. R. Jin, A. R. Mackintosh, E. Gu, R. A. Pethrick, and M. D. Dawson, Opt. Express 16, 18933 (2008).

27. S. Nizamoglu, T. Ozel, E. Sari, and H. V. Demir, Nanotechnology 18, 065709 (2007).

28. A. Žukauskas, R. Vaicekauskas, F. Ivanauskas, R. Gaska, and M. S. Shur, Appl. Phys. Lett. 80, 234 (2002).

29. Y. Ohno, Proc. SPIE 5530, 88 (2004).

30. Y. Ohno, Opt. Eng. 44, 111302 (2005).

31. W. Davis and Y. Ohno, Proc. SPIE 5941, $59411 \mathrm{G}$ (2005).

32. S. M. Berman, D. J. Jewett, G. Fein, G. Saika, F. Ashford, Lighting Res. Technol. 22, 37 (1990). 